\title{
Explicit iteration to Hadamard fractional integro-differential equations on infinite domain
}

\author{
Guotao Wang ${ }^{1 *}$, Ke Pei $^{1}$ and Dumitru Baleanu ${ }^{2,3}$
}

\section{"Correspondence:}

wgt2512@163.com

${ }^{1}$ School of Mathematics, Shanxi

Normal University, Linfen, Shanxi

041004, People's Republic of China

Full list of author information is

available at the end of the article

\section{Springer}

\begin{abstract}
This paper investigates the existence of the unique solution for a Hadamard fractional integral boundary value problem of a Hadamard fractional integro-differential equation with the monotone iterative technique. Two examples to illustrate our result are given.
\end{abstract}

Keywords: Hadamard derivative; Hadamard integro-differential boundary conditions; monotone iterative; infinite interval

\section{Introduction}

Fractional differential equations are becoming more and more popular recently in several journals and books due to their applications in a number of fields such as physics, biophysics, mechanical systems, electrical-analytical, and thermal systems [1-6]. For some recent development of this topic, see for example [7-13] and the references therein.

In 1892 [14], Hadamard presented a concept of fractional derivatives, which is different from Caputo and Riemann-Liouville type fractional derivatives and involves a logarithmic function of an arbitrary exponent in the integral kernel. It is significant that the study of Hadamard type fractional differential equations is still in its infancy and deserves further study. A detailed presentation of Hadamard fractional derivative is available in [3] and [15-23].

As was pointed out in [22], Hadamard's construction is more appropriate for problems on half axes. In this situation, we consider the following Hadamard fractional integrodifferential equations with Hadamard fractional integral boundary conditions on an infinite interval:

$$
\left\{\begin{array}{l}
{ }^{H} D^{\gamma} u(t)+f\left(t, u(t),{ }^{H} I^{q} u(t)\right)=0, \quad 2<\gamma<3, t \in(1,+\infty), \\
u(1)=u^{\prime}(1)=0, \quad{ }^{H} D^{\gamma-1} u(\infty)=\sum_{i=1}^{m} \lambda_{i}{ }^{H} I^{\beta_{i}} u(\eta),
\end{array}\right.
$$

where ${ }^{H} D^{\gamma}$ denotes Hadamard fractional derivative of order $\gamma, \eta \in(1, \infty)$, and ${ }^{H} I^{(\cdot)}$ is the Hadamard fractional integral, $q, \beta_{i}>0(i=1,2, \ldots, m), \lambda_{i} \geq 0(i=1,2, \ldots, m)$ are given constants and $\gamma, \eta, \beta_{i}, \lambda_{i}$ satisfy $\Gamma(\gamma)>\sum_{i=1}^{m} \frac{\lambda_{i} \Gamma(\gamma)}{\Gamma\left(\gamma+\beta_{i}\right)}(\log \eta)^{\gamma+\beta_{i}-1}$.

(c) Wang et al. 2016. This article is distributed under the terms of the Creative Commons Attribution 4.0 International License (http://creativecommons.org/licenses/by/4.0/), which permits unrestricted use, distribution, and reproduction in any medium, provided you give appropriate credit to the original author(s) and the source, provide a link to the Creative Commons license, and indicate if changes were made. 
We recall that the monotone iterative technique represents a powerful tool for seeking the solution of a nonlinear problem. For more details as regards the application of this method in fractional differential equations, see [24-38] and the references therein.

We organize the rest of our manuscript as follows: In Section 2, we show some useful preliminaries and the key lemmas that are used in subsequent part of the manuscript. Then, in Section 3, the main results and proofs are provided. Section 4, exhibits two examples to illustrate our main results.

\section{Preliminaries}

Below, we will present some useful definitions and related lemmas.

Define

$$
E=\left\{u \in C([1, \infty), \mathbb{R}): \sup _{t \in[1, \infty)} \frac{|u(t)|}{1+(\log t)^{\gamma-1}}<\infty\right\}
$$

then $E$ denotes a Banach space equipped with norm $\|u\|_{E}=\sup _{t \in[1, \infty)} \frac{|u(t)|}{1+(\log t)^{\gamma-1}}$.

Denote

$$
\Omega=\Gamma(\gamma)-\sum_{i=1}^{m} \frac{\lambda_{i} \Gamma(\gamma)}{\Gamma\left(\gamma+\beta_{i}\right)}(\log \eta)^{\gamma+\beta_{i}-1}
$$

obviously $\Omega>0$.

Definition 2.1 [3] For a function $g$, the Hadamard fractional integral of order $\gamma$ has the following form:

$$
{ }^{H} I^{\gamma} g(t)=\frac{1}{\Gamma(\gamma)} \int_{1}^{t}\left(\log \frac{t}{s}\right)^{\gamma-1} \frac{g(s)}{s} d s, \quad \gamma>0,
$$

provided the integral exists.

Definition 2.2 [3] The Hadamard fractional derivative of fractional order $\gamma$ for a function $g:[1, \infty) \rightarrow \mathbb{R}$ has the following form:

$$
{ }^{H} D^{\gamma} g(t)=\frac{1}{\Gamma(n-\gamma)}\left(t \frac{d}{d t}\right)^{n} \int_{1}^{t}\left(\log \frac{t}{s}\right)^{n-\gamma-1} \frac{g(s)}{s} d s, \quad n-1<\gamma<n, n=[\gamma]+1,
$$

where $[\gamma]$ means the integer part of the real number $\gamma$ and $\log (\cdot)=\log _{e}(\cdot)$.

Lemma 2.1 [3] If $a, \gamma, \beta>0$ then

$$
{ }^{H} I_{a}^{\gamma}\left(\log \left(\frac{t}{a}\right)^{\beta-1}\right)(x)=\frac{\Gamma(\beta)}{\Gamma(\beta+\gamma)}\left(\log \frac{x}{a}\right)^{\beta+\gamma-1} .
$$

Lemma 2.2 [3] If $a, \gamma, \beta>0$ then

$$
{ }^{H} D_{a}^{\gamma}\left(\log \left(\frac{t}{a}\right)^{\beta-1}\right)(x)=\frac{\Gamma(\beta)}{\Gamma(\beta-\gamma)}\left(\log \frac{x}{a}\right)^{\beta-\gamma-1} .
$$


Lemma 2.3 [3] Given $\gamma>0$ and $x \in C[1, \infty) \cap L^{1}[1, \infty)$, then the solution of the Hadamard fractional differential equation ${ }^{H} D^{\gamma} x(t)=0$ is

$$
x(t)=\sum_{i=1}^{n} c_{i}(\log t)^{\gamma-i}
$$

and

$$
{ }^{H} I^{\gamma H} D^{\gamma} x(t)=x(t)+\sum_{i=1}^{n} c_{i}(\log t)^{\gamma-i}
$$

where $c_{i} \in \mathbb{R}, i=1,2, \ldots, n$, and $n-1<\gamma<n$.

Lemma 2.4 Let $h \in C[1, \infty)$ with $0<\int_{1}^{\infty} h(s) \frac{d s}{s}<\infty$, then the Hadamard fractional integral boundary value problem

$$
\left\{\begin{array}{l}
{ }^{H} D^{\gamma} u(t)+h(t)=0, \quad 2<\gamma<3, t \in(1,+\infty) \\
u(1)=u^{\prime}(1)=0, \quad{ }^{H} D^{\gamma-1} u(\infty)=\sum_{i=1}^{m} \lambda_{i}{ }^{H} I^{\beta_{i}} u(\eta)
\end{array}\right.
$$

has the unique solution

$$
u(t)=\int_{1}^{\infty} G(t, s) h(s) \frac{d s}{s}
$$

where

$$
G(t, s)=g(t, s)+\sum_{i=1}^{m} \frac{\lambda_{i}(\log t)^{\gamma-1}}{\Omega \Gamma\left(\gamma+\beta_{i}\right)} g_{i}(\eta, s),
$$

and

$$
\begin{aligned}
& g(t, s)=\frac{1}{\Gamma(\gamma)} \begin{cases}(\log t)^{\gamma-1}-\left(\log \left(\frac{t}{s}\right)\right)^{\gamma-1}, & 1 \leq s \leq t<\infty, \\
(\log t)^{\gamma-1}, & 1 \leq t \leq s<\infty,\end{cases} \\
& g_{i}(\eta, s)= \begin{cases}(\log \eta)^{\gamma+\beta_{i}-1}-\left(\log \left(\frac{\eta}{s}\right)\right)^{\gamma+\beta_{i}-1}, & 1 \leq s \leq \eta<\infty, \\
(\log \eta)^{\gamma+\beta_{i}-1}, & 1 \leq \eta \leq s<\infty .\end{cases}
\end{aligned}
$$

Proof We apply the Hadamard fractional integral of order $\gamma$ to

$$
{ }^{H} D^{\gamma} u(t)+h(t)=0
$$

and we conclude that

$$
u(t)=c_{1}(\log t)^{\gamma-1}+c_{2}(\log t)^{\gamma-2}+c_{3}(\log t)^{\gamma-3}-\frac{1}{\Gamma(\gamma)} \int_{1}^{t}\left(\log \frac{t}{s}\right)^{\gamma-1} h(s) \frac{d s}{s},
$$

where $c_{1}, c_{2}, c_{3} \in \mathbb{R}$. 
Using the fact that $u(1)=u^{\prime}(1)=0$, we conclude that $c_{2}=c_{3}=0$. Thus,

$$
u(t)=c_{1}(\log t)^{\gamma-1}-\frac{1}{\Gamma(\gamma)} \int_{1}^{t}\left(\log \frac{t}{s}\right)^{\gamma-1} h(s) \frac{d s}{s} .
$$

Lemma 2.2 implies that

$$
{ }^{H} D^{\gamma-1} u(t)=c_{1} \Gamma(\gamma)-\int_{1}^{t} h(s) \frac{d s}{s} .
$$

Thus, the condition

$$
{ }^{H} D^{\gamma-1}(\infty)=\sum_{i=1}^{m} \lambda_{i}{ }^{H} I^{\beta_{i}} u(\eta)
$$

leads to

$$
c_{1}=\frac{1}{\Omega}\left(\int_{1}^{\infty} h(s) \frac{d s}{s}-\sum_{i=1}^{m} \frac{\lambda_{i}}{\Gamma\left(\gamma+\beta_{i}\right)} \int_{1}^{\eta}\left(\log \frac{\eta}{s}\right)^{\gamma+\beta_{i}-1} h(s) \frac{d s}{s}\right),
$$

where $\Omega$ is defined by (2.2). Substituting $c_{2}=c_{3}=0$ and (2.13) into (2.10), we get the unique solution of the Hadamard fractional integral boundary value problem (2.5)

$$
\begin{aligned}
u(t)= & \frac{(\log t)^{\gamma-1}}{\Omega}\left(\int_{1}^{\infty} h(s) \frac{d s}{s}\right. \\
& \left.-\sum_{i=1}^{m} \frac{\lambda_{i}}{\Gamma\left(\gamma+\beta_{i}\right)} \int_{1}^{\eta}\left(\log \frac{\eta}{s}\right)^{\gamma+\beta_{i}-1} h(s) \frac{d s}{s}\right) \\
& -\frac{1}{\Gamma(\gamma)} \int_{1}^{t}\left(\log \frac{t}{s}\right)^{\gamma-1} h(s) \frac{d s}{s} \\
= & \int_{1}^{\infty} g(t, s) h(s) \frac{d s}{s}+\sum_{i=1}^{m} \frac{\lambda_{i}(\log t)^{\gamma-1}}{\Omega \Gamma\left(\gamma+\beta_{i}\right)} \int_{1}^{\infty} g_{i}(\eta, s) h(s) \frac{d s}{s} \\
= & \int_{1}^{\infty} G(t, s) h(s) \frac{d s}{s} .
\end{aligned}
$$

The proof is finished.

Lemma 2.5 The Green's function $G(t, s)$ defined by (2.7) has the following properties:

$\left(A_{1}\right): G(t, s)$ is continuous and $G(t, s) \geq 0$ for $(t, s) \in[1, \infty) \times[1, \infty)$.

$\left(A_{2}\right): \frac{G(t, s)}{1+(\log t)^{\gamma-1}} \leq \frac{1}{\Gamma(\gamma)}+\sum_{i=1}^{m} \frac{\lambda_{i} g_{i}(\eta, s)}{\Omega \Gamma\left(\gamma+\beta_{i}\right)}$ for all $s, t \in[1, \infty)$.

Proof Since $\left(A_{1}\right)$ it is easy to prove, we do not present it but only prove the property $\left(A_{2}\right)$.

For $\forall s, t \in[1, \infty)$,

$$
\frac{G(t, s)}{1+(\log t)^{\gamma-1}}=\frac{1}{1+(\log t)^{\gamma-1}}\left[g(t, s)+\sum_{i=1}^{m} \frac{\lambda_{i}(\log t)^{\gamma-1} g_{i}(\eta, s)}{\Omega \Gamma\left(\gamma+\beta_{i}\right)}\right]
$$




$$
\begin{aligned}
& \leq \frac{1}{\Gamma(\gamma)}+\sum_{i=1}^{m} \frac{\lambda_{i}(\log t)^{\gamma-1} g_{i}(\eta, s)}{\Omega \Gamma\left(\gamma+\beta_{i}\right)\left(1+(\log t)^{\gamma-1}\right)} \\
& \leq \frac{1}{\Gamma(\gamma)}+\sum_{i=1}^{m} \frac{\lambda_{i} g_{i}(\eta, s)}{\Omega \Gamma\left(\gamma+\beta_{i}\right)}
\end{aligned}
$$

We present the following conditions for the sake of convenience:

$\left(C_{1}\right)$ : There exist two positive functions $p(t)$ and $q(t)$ such that

$$
\begin{aligned}
& \Lambda=\int_{1}^{\infty}\left[1+(\log t)^{\gamma-1}\right]\left[p(t)+\frac{q(t)(\log t)^{q}}{\Gamma(q)}\right] \frac{d t}{t}<\infty \\
& |f(t, u, v)-f(t, \bar{u}, \bar{v})| \leq p(t)|u-\bar{u}|+q(t)|v-\bar{v}|, \quad t \in[1, \infty), u, v, \bar{u}, \bar{v} \in \mathbb{R} .
\end{aligned}
$$

$\left(C_{2}\right)$ :

$$
\lambda=\int_{1}^{\infty}|f(t, 0,0)| \frac{d t}{t}<\infty
$$

Lemma 2.6 If $\left(C_{1}\right),\left(C_{2}\right)$ hold, then for any $u \in E$

$$
\int_{1}^{\infty} \mid f\left(t, u(t),{ }^{H} I^{q} u(t) \mid \frac{d t}{t} \leq \Lambda\|u\|_{E}+\lambda\right.
$$

Proof For any $u \in E$, taking $\bar{u}=0$, then ${ }^{H} I^{q} \bar{u}=0$. Thus, by condition $\left(C_{1}\right)$ we have

$$
\begin{aligned}
\left|f\left(t, u(t),{ }^{H} I^{q} u(t)\right)\right| \leq & p(t)|u(t)|+\left.q(t)\right|^{H} I^{q} u(t)|+| f(t, 0,0) \mid \\
\leq & p(t)\left[1+(\log t)^{\gamma-1}\right] \frac{|u(t)|}{1+(\log t)^{\gamma-1}} \\
& +q(t) \frac{1}{\Gamma(q)} \int_{1}^{t}\left(\log \frac{t}{s}\right)^{q-1} \frac{|u(s)|}{s} d s+|f(t, 0,0)| \\
\leq & p(t)\left[1+(\log t)^{\gamma-1}\right]\|u\|_{E} \\
& +q(t) \frac{1+(\log t)^{\gamma-1}}{\Gamma(q)} \\
& \times \int_{1}^{t} \frac{\left(\log \frac{t}{s}\right)^{q-1}}{s} \frac{|u(s)|}{1+(\log s)^{\gamma-1}} \frac{1+(\log s)^{\gamma-1}}{1+(\log t)^{\gamma-1}} d s \\
& +|f(t, 0,0)| \\
\leq & p(t)\left[1+(\log t)^{\gamma-1}\right]\|u\|_{E} \\
& +q(t) \frac{1+(\log t)^{\gamma-1}}{\Gamma(q)}\|u\|_{E} \int_{1}^{t} \frac{(\log t)^{q-1}}{s} d s+|f(t, 0,0)| \\
\leq & p(t)\left[1+(\log t)^{\gamma-1}\right]\|u\|_{E}+q(t) \frac{1+(\log t)^{\gamma-1}}{\Gamma(q)}(\log t)^{q}\|u\|_{E} \\
& +|f(t, 0,0)|,
\end{aligned}
$$


from which, combined with $\left(C_{1}\right)$ and $\left(C_{2}\right)$, we can obtain

$$
\begin{aligned}
\int_{1}^{\infty}\left|f\left(t, u(t),{ }^{H} I^{q} u(t)\right)\right| \frac{d t}{t} \leq & \int_{1}^{\infty} p(t)\left[1+(\log t)^{\gamma-1}\right]\|u\|_{E} \frac{d t}{t} \\
& +\int_{1}^{\infty} q(t) \frac{1+(\log t)^{\gamma-1}}{\Gamma(q)}(\log t)^{q}\|u\|_{E} \frac{d t}{t} \\
& +\int_{1}^{\infty}|f(t, 0,0)| \frac{d t}{t} \\
= & \Lambda\|u\|_{E}+\lambda .
\end{aligned}
$$

The proof is done.

\section{Main results}

Theorem 3.1 Suppose that the conditions $\left(C_{1}\right)$ and $\left(C_{2}\right)$ hold. Let

$$
w=\Lambda\left(\frac{1}{\Gamma(\gamma)}+\sum_{i=1}^{m} \frac{\lambda_{i} g_{i}(\eta, s)}{\Omega \Gamma\left(\gamma+\beta_{i}\right)}\right)<1 .
$$

Then the Hadamard fractional integral boundary value problem (1.1) admits an unique solution $\widetilde{u}(t)$ in $E$. In addition, there exists a monotone iterative sequence $u_{n}(t)$ such that $u_{n}(t) \rightarrow \widetilde{u}(t)(n \rightarrow \infty)$ uniformly on any finite sub-interval of $[1, \infty)$, where

$$
u_{n}(t)=\int_{1}^{\infty} G(t, s) f\left(s, u_{n-1}(s),{ }^{H} I^{q} u_{n-1}(s)\right) \frac{d s}{s} .
$$

Furthermore, there exists an error estimate for the approximating sequence

$$
\left\|u_{n}-\widetilde{u}\right\|_{E} \leq \frac{w^{n}}{1-w}\left\|u_{1}-u_{0}\right\|_{E} \quad(n=1,2, \ldots)
$$

Proof Define the operator $T$ by

$$
(T u)(t)=\int_{1}^{\infty} G(t, s) f\left(s, u(s),{ }^{H} I^{q} u(s)\right) \frac{d s}{s} .
$$

By Lemma 2.4, the Hadamard fractional integral boundary value problem (1.1) possesses a solution $u$ iff $u$ is a solution of $u=T u$.

First, for any $t \in[1, \infty)$, by Lemma 2.5 and Lemma 2.6, we have

$$
\begin{aligned}
\frac{|(T u)(t)|}{1+(\log t)^{\gamma-1}} & \leq \int_{1}^{\infty} \frac{G(t, s)}{1+(\log t)^{\gamma-1}}\left|f\left(s, u(s),{ }^{H} I^{q} u(s)\right)\right| \frac{d s}{s} \\
& \leq\left(\frac{1}{\Gamma(\gamma)}+\sum_{i=1}^{m} \frac{\lambda_{i} g_{i}(\eta, s)}{\Omega \Gamma\left(\gamma+\beta_{i}\right)}\right)\left[\Lambda\|u\|_{E}+\lambda\right] \\
& =w\|u\|_{E}+k .
\end{aligned}
$$

This means

$$
\|T u\|_{E} \leq w\|u\|_{E}+k, \quad \forall t \in[1, \infty)
$$


where $w$ is defined in (3.1) and

$$
k=\lambda\left(\frac{1}{\Gamma(\gamma)}+\sum_{i=1}^{m} \frac{\lambda_{i} g_{i}(\eta, s)}{\Omega \Gamma\left(\gamma+\beta_{i}\right)}\right) .
$$

In addition, for any $u, \bar{u} \in E$, we have

$$
\begin{aligned}
\frac{|(T u)(t)-(T \bar{u})(t)|}{1+(\log t)^{\gamma-1} \leq} & \int_{1}^{\infty} \frac{G(t, s)}{1+(\log t)^{\gamma-1}} \mid f\left(s, u(s),{ }^{H} I^{q} u(s)-f\left(s, \bar{u}(s),{ }^{H} I^{q} \bar{u}(s)\right) \mid \frac{d s}{s}\right. \\
\leq & \int_{1}^{\infty} \frac{G(t, s)}{1+(\log t)^{\gamma-1}}\left[p(s)|u(s)-\bar{u}(s)|+\left.q(s)\right|^{H} I^{q} u(s)-{ }^{H} I^{q} \bar{u}(s) \mid\right] \frac{d s}{s} \\
\leq & \int_{1}^{\infty} \frac{G(t, s)}{1+(\log t)^{\gamma-1}} p(s)\left[1+(\log s)^{\gamma-1}\right] \frac{|u(s)-\bar{u}(s)|}{1+(\log s)^{\gamma-1}} d s \\
& +\left.\int_{1}^{\infty} \frac{G(t, s)}{1+(\log t)^{\gamma-1}} q(s)\right|^{H} I^{q} u(s)-{ }^{H} I^{q} \bar{u}(s) \mid \frac{d s}{s} \\
\leq & \int_{1}^{\infty} \frac{G(t, s)}{1+(\log t)^{\gamma-1}} p(s)\left[1+(\log s)^{\gamma-1}\right]\|u-\bar{u}\|_{E} d s \\
& +\int_{1}^{\infty} \frac{G(t, s)}{1+(\log t)^{\gamma-1}} q(s) \frac{\left[1+(\log s)^{\gamma-1}\right](\log s)^{\gamma}}{\Gamma(\gamma)}\|u-\bar{u}\|_{E} \frac{d s}{s} \\
\leq & \left(\frac{1}{\Gamma(\gamma)}+\sum_{i=1}^{m} \frac{\lambda_{i} g_{i}(\eta, s)}{\Omega \Gamma\left(\gamma+\beta_{i}\right)}\right) \\
& \times \int_{1}^{\infty}\|u-\bar{u}\|_{E}\left[1+(\log s)^{\gamma-1}\right]\left[p(s)+\frac{q(s)(\log s)^{\gamma}}{\Gamma(\gamma)}\right] \frac{d s}{s} \\
\leq & \left(\frac{1}{\Gamma(\gamma)}+\sum_{i=1}^{m} \frac{\lambda_{i} g_{i}(\eta, s)}{\Omega \Gamma\left(\gamma+\beta_{i}\right)}\right) \Lambda\|u-\bar{u}\|_{E} \\
= & w\|u-\bar{u}\|_{E} .
\end{aligned}
$$

Then we get

$$
\|T u-T \bar{u}\|_{E} \leq w\|u-\bar{u}\|_{E}, \quad \forall u, \bar{u} \in E .
$$

Through the Banach fixed point theorem, we can ensure that $T$ has a unique fixed point $\tilde{u}$ in $E$. That is, (1.1) admits a unique solution $\tilde{u}$ in $E$. In addition, for any $u_{0} \in E,\left\|u_{n}-\widetilde{u}\right\|_{E} \rightarrow 0$ as $n \rightarrow \infty$, where $u_{n}=T u_{n-1}(n=1,2, \ldots)$.

From (3.9), we have

$$
\left\|u_{n}-u_{n-1}\right\|_{E} \leq w^{n-1}\left\|u_{1}-u_{0}\right\|_{E}
$$

and

$$
\begin{aligned}
\left\|u_{n}-u_{j}\right\|_{E} & \leq\left\|u_{n}-u_{n-1}\right\|_{E}+\left\|u_{n-1}-u_{n-2}\right\|_{E}+\cdots+\left\|u_{j+1}-u_{j}\right\|_{E} \\
& \leq \frac{w^{n}\left(1-w^{n-j}\right)}{1-w}\left\|u_{1}-u_{0}\right\|_{E} .
\end{aligned}
$$


Letting $n \rightarrow \infty$ on both sides of (3.11), we conclude that

$$
\left\|u_{n}-\widetilde{u}\right\|_{E} \leq \frac{w^{n}}{1-w}\left\|u_{1}-u_{0}\right\|_{E}
$$

\section{Example}

Example 4.1 In the following we discuss the Hadamard fractional integral boundary value problem

$$
\left\{\begin{array}{c}
{ }^{H} D^{\frac{5}{2}} u(t)+\frac{e^{-3 t} t}{1+(\log t)^{\frac{3}{2}}} \cos \left(3 t^{2}\right. \\
+u(t))+\frac{3 \sqrt{\pi} e^{-3 t} t}{8\left[1+(\log t)^{\frac{3}{2}}\right](\log t)^{\frac{5}{2}}} \arctan \left({ }^{H} I^{\frac{5}{2}} u(t)\right)=0 \\
u(1)=u^{\prime}(1)=0, \quad{ }^{H} D^{\frac{3}{2}} u(+\infty)=\lambda_{1}{ }^{H} I^{\beta_{1}} u(\eta)
\end{array}\right.
$$

where $\gamma=\frac{5}{2}, m=1, q=\frac{5}{2}$, and $\lambda_{1}, \beta_{1}, \eta$ satisfy $\left(\lambda_{1} \geq 0, \beta_{1}>0, \eta>1\right)$

$$
0<\frac{\Gamma\left(\frac{1}{2}+\beta_{1}\right)}{3 \Gamma\left(\frac{1}{2}+\beta_{1}\right)-4 \lambda_{1}(\log \eta)^{\frac{3}{2}+\beta_{1}}}<\frac{e^{3} \sqrt{\pi}}{2}
$$

(see Figure 1).

For example, we can take $\lambda_{1}=\frac{1}{10}, \beta_{1}=\frac{3}{2}, \eta=\frac{5}{2}$,

$$
\begin{aligned}
& \left|f\left(t, u,{ }^{H} I^{\frac{5}{2}} u(t)\right)-f\left(t, \bar{u},{ }^{H} I^{\frac{5}{2}} \bar{u}(t)\right)\right| \\
& \leq \frac{e^{-3 t} t}{1+(\log t)^{\frac{3}{2}}}\left|\cos \left(3 t^{2}+u(t)\right)-\cos \left(3 t^{2}+\bar{u}(t)\right)\right| \\
& \quad+\frac{3 \sqrt{\pi} e^{-3 t} t}{8\left[1+(\log t)^{\frac{3}{2}}\right](\log t)^{\frac{5}{2}}}\left|\arctan \left({ }^{H} I^{\frac{5}{2}} u(t)\right)-\arctan \left({ }^{H} I^{\frac{5}{2}} \bar{u}(t)\right)\right|
\end{aligned}
$$

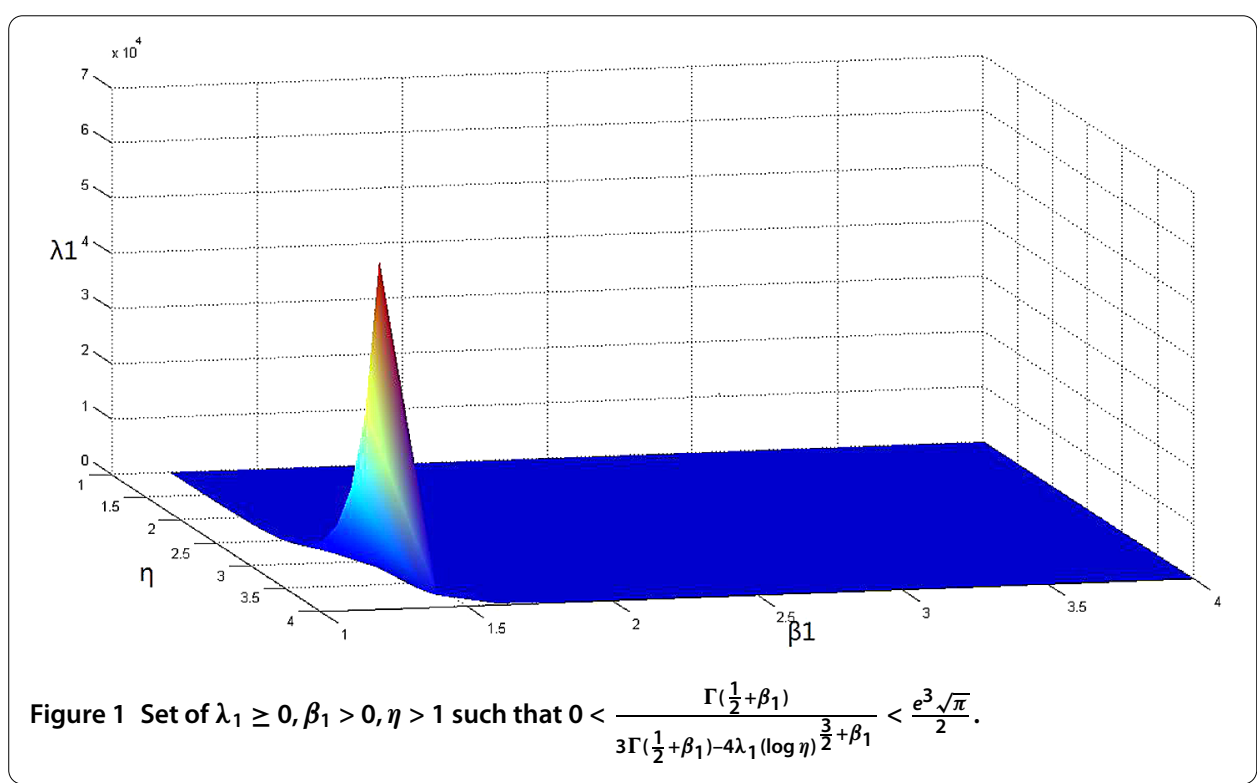




$$
\begin{aligned}
\leq & \frac{e^{-3 t} t}{1+(\log t)^{\frac{3}{2}}}|u(t)-\bar{u}(t)| \\
& +\left.\frac{3 \sqrt{\pi} e^{-3 t} t}{8\left[1+(\log t)^{\frac{3}{2}}\right](\log t)^{\frac{5}{2}}}\right|^{H} I^{\frac{5}{2}} u(t)-{ }^{H} I^{\frac{5}{2}} \bar{u}(t) \mid
\end{aligned}
$$

Since $p(t)=\frac{e^{-3 t} t}{1+(\log t)^{\frac{3}{2}}}$ and $q(t)=\frac{3 \sqrt{\pi} e^{-3 t} t}{8\left[1+(\log t)^{\frac{3}{2}}\right](\log t)^{\frac{5}{2}}}$, we can show that

$$
\begin{aligned}
\Lambda & =\int_{1}^{\infty}\left[1+(\log t)^{\frac{3}{2}}\right]\left[\frac{e^{-3 t} t}{1+(\log t)^{\frac{3}{2}}}+\frac{3 \sqrt{\pi} e^{-3 t} t(\log t)^{\frac{5}{2}}}{8\left[1+(\log t)^{\frac{3}{2}}\right](\log t)^{\frac{5}{2}} \Gamma\left(\frac{5}{2}\right)}\right] \frac{d t}{t} \\
& =\frac{1}{2 e^{3}}<\infty, \\
\lambda & =\int_{1}^{\infty}|f(t, 0,0)| \frac{d t}{t}=\int_{1}^{\infty} e^{-3 t} d t=\frac{1}{3 e^{3}}<\infty .
\end{aligned}
$$

Then $\left(C_{1}\right)$ and $\left(C_{2}\right)$ hold. At last, by a simple computation, we have

$$
\begin{aligned}
& \Omega=\Gamma(\gamma)-\frac{\lambda_{1} \Gamma(\gamma)}{\Gamma\left(\gamma+\beta_{1}\right)}(\log \eta)^{\gamma+\beta_{1}-1}=\frac{3 \sqrt{\pi}}{4}-\frac{\sqrt{\pi} \lambda_{1}(\log \eta)^{\frac{3}{2}+\beta_{1}}}{\Gamma\left(\frac{1}{2}+\beta_{1}\right)}>\frac{1}{2 e^{3}}>0, \\
& w=\Lambda\left(\frac{1}{\Gamma(\gamma)}+\frac{\lambda_{1} g_{1}(\eta, s)}{\Omega \Gamma\left(\gamma+\beta_{i}\right)}\right)=\frac{2}{e^{3} \sqrt{\pi}}\left(\frac{\Gamma\left(\frac{1}{2}+\beta_{1}\right)}{3 \Gamma\left(\frac{1}{2}+\beta_{1}\right)-4 \lambda_{1}(\log \eta)^{\frac{3}{2}+\beta_{1}}}\right)<1 .
\end{aligned}
$$

As a result, the conditions of Theorem 3.1 hold. Thus, the conclusion of Theorem 3.1 implies that (4.1) possesses a unique solution.

Example 4.2 Let us discuss the following Hadamard fractional integral boundary value problem:

$$
\left\{\begin{array}{l}
{ }^{H} D^{\frac{9}{4}} u(t)+f\left(t, u(t),{ }^{H} I^{\frac{9}{2}} u(t)\right)=0, \\
u(1)=u^{\prime}(1)=0, \quad{ }^{H} D^{\frac{9}{4}} u(+\infty)=\sum_{i=1}^{3} \lambda_{i}{ }^{H} I^{\beta_{i}} u(\eta)
\end{array}\right.
$$

here

$$
f\left(t, u(t),{ }^{H} I^{\frac{9}{2}} u(t)\right)=\frac{\sin \left(t^{2}+u(t)\right)}{16\left(1+t^{2}\right)\left[1+(\log t)^{\frac{5}{4}}\right]}+\frac{{ }^{H} I^{\frac{9}{2}} u(t)-\sin \left({ }^{H} I^{\frac{9}{2}} u(t)\right) \cos \left({ }^{H} I^{\frac{9}{2}} u(t)\right)}{128\left(1+t^{2}\right)\left[1+(\log t)^{\frac{5}{4}}\right](\log t)^{\frac{9}{2}}} .
$$

Take $\gamma=\frac{9}{4}, m=3, q=\frac{9}{2}, \eta=\frac{9}{4}, \lambda_{1}=\frac{1}{10}, \beta_{1}=\frac{3}{4}, \lambda_{2}=\frac{\sqrt{\pi}}{2}, \beta_{2}=\frac{7}{4}, \lambda_{3}=4$, and $\beta_{3}=\frac{11}{4}$.

The function $f$ satisfies the inequality

$$
\begin{aligned}
& \left|f\left(t, u,{ }^{H} I^{\frac{9}{2}} u(t)\right)-f\left(t, \bar{u},{ }^{H} I^{\frac{9}{2}} \bar{u}(t)\right)\right| \\
& \leq \leq \frac{1}{16\left(1+t^{2}\right)\left[1+(\log t)^{\frac{5}{4}}\right]}|u(t)-\bar{u}(t)| \\
& \quad+\left.\frac{1}{64\left(1+t^{2}\right)\left[1+(\log t)^{\frac{5}{4}}\right](\log t)^{\frac{9}{2}}}\right|^{H} I^{\frac{9}{2}} u(t)-{ }^{H} I^{\frac{9}{2}} \bar{u}(t) \mid .
\end{aligned}
$$




$$
\begin{aligned}
& \text { Since } p(t)=\frac{1}{16\left(1+t^{2}\right)\left[1+(\log t)^{\frac{5}{4}}\right]} \text { and } q(t)=\frac{1}{64\left(1+t^{2}\right)\left[1+(\log t)^{\frac{5}{4}}\right](\log t)^{\frac{9}{2}}} \text {, we can show that } \\
& \begin{aligned}
\Lambda=\int_{1}^{\infty}\left[1+(\log t)^{\frac{5}{4}}\right] \\
\quad \times\left[\frac{1}{16\left(1+t^{2}\right)\left[1+(\log t)^{\frac{5}{4}}\right]}+\frac{(\log t)^{\frac{9}{2}}}{64 \Gamma\left(\frac{9}{2}\right)\left(1+t^{2}\right)\left[1+(\log t)^{\frac{5}{4}}\right](\log t)^{\frac{9}{2}}}\right] \frac{d t}{t} \\
<\frac{\pi}{32}<\infty, \\
\lambda=\int_{1}^{\infty}|f(t, 0,0)| \frac{d t}{t}<\int_{1}^{\infty} \frac{1}{4\left(1+t^{2}\right)} d t=\frac{\pi}{64}<\infty .
\end{aligned}
\end{aligned}
$$

Then $\left(C_{1}\right)$ and $\left(C_{2}\right)$ hold. At last, by a simple computation, we have

$$
\begin{aligned}
& \Omega=\Gamma\left(\frac{9}{4}\right)-\sum_{i=1}^{3} \frac{\lambda_{i} \Gamma\left(\frac{9}{4}\right)}{\Gamma\left(\frac{9}{4}+\beta_{i}\right)}(\log \eta)^{\frac{9}{4}+\beta_{i}-1} \approx 0.8562>0, \\
& w=\Lambda\left(\frac{1}{\Gamma\left(\frac{9}{4}\right)}+\sum_{i=1}^{3} \frac{\lambda_{i} g_{i}(\eta, s)}{\Omega \Gamma\left(\frac{9}{4}+\beta_{i}\right)}\right) \approx 0.0452<1 .
\end{aligned}
$$

Thus, by the application of Theorem 3.1 the Hadamard fractional integral boundary value problem (4.3) admits an unique solution.

\section{Competing interests}

The authors declare that they have no competing interests.

\section{Authors' contributions}

All authors contributed equally to the writing of this paper. All authors read and approved the final manuscript.

\section{Author details}

'School of Mathematics, Shanxi Normal University, Linfen, Shanxi 041004, People's Republic of China. ${ }^{2}$ Department of Mathematics, Faculty of Art and Sciences, Çankaya University, Balgat, 06530, Turkey. ${ }^{3}$ Institute of Space Sciences, Magurele-Bucharest, Romania.

\section{Acknowledgements}

Partially supported by National Natural Science Foundation of China (No. 11501342) and the Scientific and Technological Innovation Programs of Higher Education Institutions in Shanxi (Nos. 2014135 and 2014136)

Received: 10 September 2016 Accepted: 9 November 2016 Published online: 23 November 2016

\section{References}

1. Podlubny, I: Fractional Differential Equations. Academic Press, San Diego (1999)

2. Magin, RL: Fractional Calculus in Bioengineering. Begell House Publisher, Inc., Connecticut (2006)

3. Kilbas, AA, Srivastava, HM, Trujillo, JJ: Theory and Applications of Fractional Differential Equations. North-Holland Mathematics Studies, vol. 204. Elsevier Science B.V., Amsterdam (2006)

4. Sabatier, J, Agrawal, OP, Machado, JAT (eds.): Advances in Fractional Calculus: Theoretical Developments and Applications in Physics and Engineering. Springer, Dordrecht (2007)

5. Lakshmikantham, V, Leela, S, Devi, JV: Theory of Fractional Dynamic Systems. Cambridge Scientific Publishers, Cambridge (2009)

6. Baleanu, D, Diethelm, K, Scalas, E, Trujillo, JJ: Fractional Calculus Models and Numerical Methods. Series on Complexity, Nonlinearity and Chaos. World Scientific, Boston (2012)

7. Zhang, X, Liu, L, Wu, Y: The uniqueness of positive solution for a singular fractional differential system involving derivatives. Commun. Nonlinear Sci. Numer. Simul. 18, 1400-1409 (2013)

8. Zhang, X, Liu, L, Wiwatanapataphee, B, Wu, Y: The eigenvalue for a class of singular p-Laplacian fractional differential equations involving the Riemann-Stieltjes integral boundary condition. Appl. Math. Comput. 235, 412-422 (2014)

9. Zhang, X, Liu, L, Wu, Y: Variational structure and multiple solutions for a fractional advection-dispersion equation. Comput. Math. Appl. 68, 1794-1805 (2014)

10. Zhang, $X, L i u, L, W u, Y$ : The uniqueness of positive solution for a fractional order model of turbulent flow in a porous medium. Appl. Math. Lett. 37, 26-33 (2014) 
11. Zhang, $X, W u, Y$, Lou, C: Nonlocal fractional order differential equations with changing-sign singular perturbation. Appl. Math. Model. 39, 6543-6552 (2015)

12. Zhang, $X, L i u, L, W u, Y$, Wiwatanapataphee, $B$ : The spectral analysis for a singular fractional differential equation with a signed measure. Appl. Math. Comput. 257, 252-263 (2015)

13. Zhang, $X, L i u, L$, Wiwatanapataphee, $B, W u, Y$ : Positive solutions of eigenvalue problems for a class of fractional differential equations with derivatives. Abstr. Appl. Anal. 2012, Article ID 512127 (2012)

14. Hadamard, J: Essai sur l'étude des fonctions données par leur developpement de Taylor. J. Math. Pures Appl. 8, 101-186 (1892)

15. Kilbas, AA: Hadamard-type fractional calculus. J. Korean Math. Soc. 38, 1191-1204 (2001)

16. Butzer, PL, Kilbas, AA, Trujillo, JJ: Compositions of Hadamard-type fractional integration operators and the semigroup property. J. Math. Anal. Appl. 269, 387-400 (2002)

17. Kilbas, AA, Trujillo, JJ: Hadamard-type integrals as G-transforms. Integral Transforms Spec. Funct. 14, 413-427 (2003)

18. Ahmad, B, Ntouyas, S: A fully Hadamard type integral boundary value problem of a coupled system of fractional differential equations. Fract. Calc. Appl. Anal. 17(2), 348-360 (2014)

19. Garra, R, Polito, F: On some operators involving Hadamard derivatives. Integral Transforms Spec. Funct. 24, 773-782 (2013)

20. Wang, J, Zhou, Y, Medved, M: Existence and stability of fractional differential equations with Hadamard derivative. Topol. Methods Nonlinear Anal. 41, 113-133 (2013)

21. $\mathrm{Ma}, \mathrm{Q}$, Wang, $\mathrm{R}$, Wang, J, Ma, Y: Qualitative analysis for solutions of a certain more generalized two-dimensional fractional differential system with Hadamard derivative. Appl. Math. Comput. 257, 436-445 (2015)

22. Aljoudi, S, Ahmad, B, Nieto, JJ, Alsaedi, A: A coupled system of Hadamard type sequential fractional differential equations with coupled strip conditions. Chaos Solitons Fractals 91, 39-46 (2016)

23. Yukunthorn, W, Ahmad, B, Ntouyas, SK, Tariboon, J: On Caputo-Hadamard type fractional impulsive hybrid systems with nonlinear fractional integral conditions. Nonlinear Anal. Hybrid Syst. 19, 77-92 (2016)

24. Lakshmikantham, V, Leela, S, Vasundhara, J: Theory of Fractional Dynamic Systems. Cambridge Academic Publishers, Cambridge (2009)

25. McRae, FA: Monotone iterative technique and existence results for fractional differential equations. Nonlinear Anal. 71, 6093-6096 (2009)

26. Zhang, S: Monotone iterative method for initial value problem involving Riemann-Liouville fractional derivatives. Nonlinear Anal. 71, 2087-2093 (2009)

27. Wei, Z, Dong, W, Che, J: Periodic boundary value problems for fractional differential equations involving a Riemann-Liouville fractional derivative. Nonlinear Anal. 73, 3232-3238 (2010)

28. Al-Refai, M, Hajji, MA: Monotone iterative sequences for nonlinear boundary value problems of fractional order. Nonlinear Anal. 74, 3531-3539 (2011)

29. Ramirez, JD, Vatsala, AS: Monotone method for nonlinear Caputo fractional boundary value problems. Dyn. Syst. Appl. 20, 73-88 (2011)

30. Wang, G: Monotone iterative technique for boundary value problems of a nonlinear fractional dif- ferential equations with deviating arguments. J. Comput. Appl. Math. 236, 2425-2430 (2012)

31. Wang, G, Agarwal, RP, Cabada, A: Existence results and the monotone iterative technique for systems of nonlinear fractional differential equations. Appl. Math. Lett. 25, 1019-1024 (2012)

32. Wang, G, Baleanu, D, Zhang, L: Monotone iterative method for a class of nonlinear fractional differential equations. Fract. Calc. Appl. Anal. 15, 244-252 (2012)

33. $\mathrm{Hu}, \mathrm{C}, \mathrm{Liu}, \mathrm{B}, \mathrm{Xie}, \mathrm{S}$ : Monotone iterative solutions for nonlinear boundary value problems of fractional differential equation with deviating arguments. Appl. Math. Comput. 222, 72-81 (2013)

34. Liu, Z, Sun, J, Szanto, I: Monotone iterative technique for Riemann-Liouville fractional integro-differential equations with advanced arguments. Results Math. 63, 1277-1287 (2013)

35. Zhang, $X, L i u, L, W u, Y, L u, Y$ : The iterative solutions of nonlinear fractional differential equations. Appl. Math. Comput. 219, 4680-4691 (2013)

36. Zhang, L, Ahmad, B, Wang, G, Agarwal, RP: Nonlinear fractional integro-differential equations on unbounded domains in a Banach space. J. Comput. Appl. Math. 249, 51-56 (2013)

37. Zhang, L, Ahmad, B, Wang, G: Successive iterations for positive extremal solutions of nonlinear fractional differential equations on a half-line. Bull. Aust. Math. Soc. 91, 116-128 (2015)

38. Wang, G: Explicit iteration and unbounded solutions for fractional integral boundary value problem on an infinite interval. Appl. Math. Lett. 47, 1-7 (2015)

\section{Submit your manuscript to a SpringerOpen ${ }^{\circ}$ journal and benefit from:}

- Convenient online submission

- Rigorous peer review

- Immediate publication on acceptance

Open access: articles freely available online

High visibility within the field

- Retaining the copyright to your article

Submit your next manuscript at $\boldsymbol{~ s p r i n g e r o p e n . c o m ~}$ 